\title{
Evaluation on Long-Term Test-Retest Reliability of the Short-Form Childhood Trauma Questionnaire in Patients with Schizophrenia
}

\author{
Zhibiao Xiang $^{1-3}$ \\ Zhening Liu ${ }^{1-3}$ \\ Hengyi $\mathrm{Cao}^{4,5}$ \\ Zhipeng $\mathrm{Wu}^{\mathrm{I}-3}$ \\ Yicheng Long ${ }^{1-3}$
}

'Department of Psychiatry, The Second Xiangya Hospital, Central South University, Changsha, Hunan, People's Republic of China; ${ }^{2}$ Mental Health Institute of Central South University, Changsha, Hunan, People's Republic of China; ${ }^{3}$ China National Clinical Research Center on Mental Disorders, Changsha, Hunan, People's Republic of China; ${ }^{4}$ Center for Psychiatric Neuroscience, Feinstein Institute for Medical Research, Hempstead, NY, USA; ${ }^{5}$ Division of Psychiatry Research, Zucker Hillside Hospital, Glen Oaks, NY, USA
Correspondence: Yicheng Long Email yichenglong@csu.edu.cn
Background: Many studies have reported an association between childhood trauma exposure and schizophrenia. Among these studies, the Short-form Childhood Trauma Questionnaire (CTQ-SF) is one of the most widely used measures of childhood trauma. However, little is known regarding the long-term reliability of the CTQ-SF, especially in patients with psychopathology.

Methods: The CTQ-SF was administered to 50 patients diagnosed with schizophrenia from a hospital in Changsha, Hunan, China. These patients were asked to re-complete the CTQ-SF when they were re-hospitalized or received outpatient treatments in the same hospital within 4 years of follow-up. Intraclass correlation coefficient (ICC) was used to assess test-retest reliability of the CTQ-SF over the intervals. Associations of the CTQ-SF with the Positive and Negative Syndrome Scale (PANSS) and Wechsler Adult Intelligence Scale (WAIS) were tested using Spearman correlation coefficients.

Results: Among the participants, $35(70.0 \%)$ patients re-completed the CTQ-SF after an interval averaging 11.26 months. Excellent test-retest reliabilities (with ICC $>0.75$ ) were found for the total CTQ-SF score $(\mathrm{ICC}=0.772)$ as well as scores of the emotional abuse $(\mathrm{ICC}=0.808)$, physical abuse $(\mathrm{ICC}=0.756)$, sexual abuse $(\mathrm{ICC}=0.877)$ and physical neglect $(\mathrm{ICC}=0.751)$ subscales. Meanwhile, a moderate test-retest reliability was found for the emotional neglect subscale ( $\mathrm{ICC}=0.538$ ). At both baseline and follow-up, no significant correlations $(p>0.05)$ were found between CTQ-SF scores and any other clinical assessments.

Conclusion: Our results suggest that CTQ-SF is reliable to assess childhood trauma exposures in schizophrenia over relatively long intervals, regardless of patients' current symptoms and states of cognition.

Keywords: childhood trauma questionnaire, childhood adversity, childhood trauma, schizophrenia, test-retest reliability

\section{Introduction}

Schizophrenia is a serious mental disorder characterized by a range of symptoms including delusions and hallucinations, ${ }^{1}$ and is associated with alterations in brain structure $^{2}$ and function. ${ }^{3,4}$ Several studies have reported an association between childhood trauma exposure and schizophrenia, in which patients with schizophrenia report higher levels of childhood trauma compared to the general population., Moreover, some of the brain alterations observed in schizophrenia have been associated with childhood trauma histories, including altered grey matter volume patterns (eg decreased hippocampus volumes $)^{7-10}$ as well as disrupted brain 
function (eg decreased connectivity and decreased stability within multiple brain areas) during rest ${ }^{4,11,12}$ or specific tasks. $^{13-16}$ These results highlight the important role of childhood trauma in the development of schizophrenia, as a part of the integrative "bio-psycho-social" model. ${ }^{5}$

In the field of childhood trauma research, it is common to assess childhood trauma by self-reported questionnaires. ${ }^{17}$ Among these questionnaires, the Short-form Childhood Trauma Questionnaire (CTQ-SF) ${ }^{18}$ is one of the most widely used and well-studied tools. ${ }^{12,19-23}$ The CTQ-SF consists of five subscales to assess five subtypes of childhood trauma: emotional abuse, physical abuse, sexual abuse, emotional neglect and physical neglect. ${ }^{18}$ Previous studies have demonstrated the validity and overall good test-retest reliability of CTQ-SF in patients with schizophrenia when tested over a period of two ${ }^{24}$ or four ${ }^{25}$ weeks.

Despite these findings, it is possible that results of retrospective self-reports such as the CTQ-SF may be influenced by memory lapses and participants' psychopathological status. ${ }^{26}$ Factors such as normal processes of memory loss in ageing, ${ }^{27,28}$ change of mood status, ${ }^{29}$ subsequent events, ${ }^{30}$ and a need to justify or understand mental illness ${ }^{31,32}$ may influence the reliability of such reports, especially during long-term follow-up. For example, previous studies have indicated that self-reported data can be biased by forgetting even with a short recall period of one month. ${ }^{33}$ Therefore, it is necessary to verify whether CTQ-SF scores are robust and reliable over longer time scales despite the influence of these various factors. However, research regarding the test-retest reliability of CTQ-SF in schizophrenia patients with longterm follow-up periods (ie, two months or longer) ${ }^{34}$ is still lacking.

Therefore, the present study aimed to examine testretest reliability of CTQ-SF in patients with schizophrenia over long terms (defined as over two months), ${ }^{34}$ in order to investigate if the CTQ-SF scores would be influenced by patients' normal processes of forgetting, as well as changed psychopathological status such as the severity of psychiatric symptoms and cognitive deficits over time. The results were anticipated to provide evidence for the validity of CTQ-SF in the studies of schizophrenia and other mental disorders.

\section{Materials and Methods}

\section{Participants}

This study included 50 schizophrenia patients, who were recruited from the Department of Psychiatry, Second
Xiangya Hospital of Central South University, Changsha, Hunan, China. The inclusion criteria for the participants were as follows: 1) met the diagnostic criteria for schizophrenia according to the Diagnostic and Statistical Manual of Mental Disorders, fifth edition (DSM-V); 2) between 14 and 35 years old; 3) no history of psychotropic substance abuse; and 4) able to understand and complete all questions of the CTQ-SF. All participants were followed-up from December, 2016 to January, 2021. During the followup, participants were excluded from the study if they had any of the following situations: 1) change in diagnosis; 2) contact was lost; and 3) participants had severe physical illnesses or extensive declines in cognition, making them unable to complete the assessments at follow-up.

\section{Procedures and Assessments}

All participants completed the CTQ-SF at baseline and were followed longitudinally. They were asked to recomplete the CTQ-SF when they were re-hospitalized or received outpatient treatments in the same hospital after a two-month or longer period within 4 years of follow-up. Besides the CTQ-SF, several other clinical assessments were also performed at both baseline and follow-up to evaluate the participants' clinical symptoms and cognitions. These assessments are described as below:

(1) CTQ-SF: ${ }^{18}$ the CTQ-SF consists of 25 clinical items which are grouped into five subscales (5 items each): emotional abuse, physical abuse, sexual abuse, emotional neglect and physical neglect to assess such five types of childhood trauma. Each of the 25 items are 5-point Likert-type questions, rated from 1 (never) to 5 (very often) according to the frequency of each event. Thus, total scores range from 25 to 125 , while the scores of each subscale range from 5 to 25 . Higher scores indicate higher levels of childhood trauma exposures. In addition to the clinical items, all participants' minimization/ denial (MD) scores were also calculated referring to a previous work. ${ }^{35}$

(2) Positive and Negative Syndrome Scale (PANSS): ${ }^{36}$ for all patients, severity of their current clinical symptoms during the past two weeks was assessed using the PANSS. This was performed through face-to-face interviews with psychiatrists who were licensed as medical practitioners in China.

(3) Wechsler Adult Intelligence Scale (WAIS): ${ }^{37}$ all participants finished the digit symbol (WAIS-DS) and information (WAIS-I) subtest of the WAIS Chinese version, which is one of the most widely used measure of 
cognitive function in China, ${ }^{2,38,39}$ to assess their current cognitive function upon completion of the CTQ-SF. The WAIS-DS and WAIS-I are assessments of two important domains of cognition: measuring processing speed and verbal comprehension, respectively. ${ }^{2,37}$

\section{Statistics}

Differences in demographic and clinical variables between different subsamples were tested using a $t$-test (for age and years of education), Chi-square test (for sex) and the Mann-Whitney $U$-test (for all clinical scales). Test-retest reliability of the CTQ-SF was evaluated by applying twoway random effects intra-class correlation coefficients (ICC). ${ }^{40}$ ICCs were calculated for both total score and each of the subscale scores of the CTQ-SF. Based on ICCs, the test-retest reliabilities can be interpreted as follows: poor reliability ( $\mathrm{ICC}<0.4$ ), moderate reliability $(0.4 \leq \mathrm{ICC}<0.6)$, good reliability $(0.6 \leq \mathrm{ICC}<0.75)$, and excellent reliability $(\mathrm{ICC} \geq 0.75){ }^{41,42}$

An additional analysis was carried out to investigate if the CTQ-SF scores were influenced by participants' current clinical symptoms or cognitions. Here, we tested possible associations between the CTQ-SF scores and PANSS/WAIS scores using the Spearman correlation coefficients. This analysis was performed at both baseline and follow-up.

All analyses were performed using the SPSS software (version 26.0) for MAC, and statistical significance was set at an alpha level of 0.05 (two-sided) for all tests.

\section{Exploratory Analysis}

An exploratory analysis was performed to investigate possible influences of follow-up interval length on results. Here, all participants were divided two subgroups considering the distribution of follow-up intervals in the current sample (averaging 11.26 months): those with an interval shorter than 12 months $(\mathrm{n}=23)$, and those with an interval longer than or equal to 12 months $(n=12)$; ICCs of the CTQ-SF scores were then calculated for each subgroup separately.

\section{Results}

\section{Participant Characteristics}

Among the initial sample of 50 patients, a total 15 participants were excluded during follow-up: 4 patients were excluded because their diagnoses were changed to depression or bipolar disorder; 6 patients were loss of contact; 5 patients had extensive declines in cognition which make them unable to re-complete the assessments. The age range of the participants who re-completed the CTQ-SF $(\mathrm{n}=35)$ were from 14 to 28 years at baseline, with 15 males and 20 females (Table 1). The test-retest intervals ranged from 2 to 41 months (11.26 months on average). There were no significant differences in age, sex, education level, CTQ-SF total score, or the scores of PANSS and WAIS at baseline between those who were followed up and those who were not (Supplementary Table 1).

\section{Changes Over Time in Clinical Variables}

Comparisons of the results of the CTQ-SF and other clinical assessments for the followed participants at baseline and follow-up are summarized in Table 1. There were no significant differences in the CTQ-SF total score ( $p=$ 0.659 ), or scores of subscales including the MD score (all $p>0.05)$. No significant differences were found in the WAIS-DS/WAIS-I scores (both $p>0.05$ ). Compared with baseline, the participants had significantly lower PANSS scores at follow-up $(p=0.045)$.

\section{Test-Retest Reliabilities}

As shown in Table 2, the Cronbach's $\alpha$ coefficient of the CTQ-SF total score was 0.871 , and the $\alpha$ coefficients for its five subscales ranged from 0.700 (physical neglect) to 0.934 (sexual abuse), suggesting a good internal consistency of the scale. The ICC of the CTQ-SF total score was 0.772 , which suggests an excellent test-retest reliability. Excellent testretest reliabilities (with $\mathrm{ICC}>0.75$ ) were also found for scores of the emotional abuse $(\mathrm{ICC}=0.808)$, physical abuse $(\mathrm{ICC}=0.756)$, sexual abuse $(\mathrm{ICC}=0.877)$ and physical neglect ( $\mathrm{ICC}=0.751$ ) subscales, while a moderate testretest reliability was found for the emotional neglect subscale $(\mathrm{ICC}=0.538)$. A poor test-retest reliability $(\mathrm{ICC}=$ 0.333 ) was found for the MD score.

\section{Relationships with Clinical Symptoms and Cognitions}

As shown in Tables 3 and 4, for both baseline and followup, no significant correlations $(p>0.05)$ were found between the CTQ-SF total score and any other clinical/ cognitive assessments including the PANSS, WAIS-I and WAIS-DS. No significant correlations $(p>0.05)$ were found between any of the CTQ-SF subscale scores (including the MD score) and any other assessments. 
Table I The Demographic and Clinical Characteristics of the Longitudinally Followed Participants

\begin{tabular}{|l|l|l|l|}
\hline & $\begin{array}{l}\text { At Baseline }(\mathbf{n}=\mathbf{3 5}), \\
\text { Mean } \mathbf{~ S D ~}\end{array}$ & $\begin{array}{l}\text { At Follow-Up }(\mathbf{n}=\mathbf{3 5}), \\
\text { Mean } \pm \text { SD }\end{array}$ & Mann-Whitney U-Tests of Differences \\
\hline Age (years) & $17.91 \pm 3.60$ & $18.76 \pm 3.84$ & $/$ \\
\hline Gender (male/female) & $15 / 20$ & $15 / 20$ & $/$ \\
\hline Education (years) & $10.74 \pm 2.24$ & $10.85 \pm 2.23$ & $/$ \\
\hline WAIS-I scores & $17.09 \pm 5.16$ & $16.91 \pm 5.79$ & $p=0.888$ \\
\hline WAIS-DS scores & $68.17 \pm 16.07$ & $72.37 \pm 19.38$ & $p=0.145$ \\
\hline PANSS scores & $61.97 \pm 21.86$ & $55.06 \pm 28.35$ & $p=0.045^{*}$ \\
\hline CTQ-SF scores & & & \\
Emotional abuse & $9.23 \pm 3.49$ & $8.37 \pm 3.20$ & $p=0.313$ \\
Physical abuse & $6.43 \pm 2.23$ & $6.26 \pm 2.02$ & $p=0.857$ \\
Sexual abuse & $6.40 \pm 2.49$ & $6.23 \pm 2.34$ & $p=0.855$ \\
Emotional neglect & $13.31 \pm 4.82$ & $9.20 \pm 2.92$ & $p=0.755$ \\
Physical neglect & $8.89 \pm 3.22$ & $43.14 \pm 9.97$ & $p=0.523$ \\
Total & $44.26 \pm 11.62$ & $0.69 \pm 0.83$ & $p=0.659$ \\
MD score & $0.65 \pm 0.76$ & $p=0.969$ \\
\hline
\end{tabular}

Note: *Indicates significance at the 0.05 level.

Table 2 Cronbach's $\alpha$ Coefficients and ICCs of the CTQ-SF and Each of Its Subscale Scores

\begin{tabular}{|l|l|l|}
\hline CTQ-SF Scores & Cronbach's $\alpha$ Coefficient & ICC (with 95\% Confidence Intervals) \\
\hline Total score & 0.871 & $0.772^{*}(0.60,0.88)$ \\
Emotional abuse & 0.861 & $0.756^{*}(0.57,0.87)$ \\
Physical abuse & 0.894 & $0.808^{*}(0.65,0.90)$ \\
Sexual abuse & 0.934 & $0.877^{*}(0.77,0.94)$ \\
Emotional neglect & 0.700 & $0.538^{*}(0.25,0.73)$ \\
Physical neglect & 0.858 & $0.75 I^{*}(0.56,0.87)$ \\
MD score & 0.500 & $0.333^{*}(0.00,0.60)$ \\
\hline
\end{tabular}

Notes: *Indicates significance at the 0.05 level.

Table 3 Correlations Between the CTQ-SF Scores and Other Clinical/Cognitive Assessments at Baseline, with Spearman's Rho and $p$ values Reported

\begin{tabular}{|l|l|l|l|}
\hline & PANSS & WAIS-DS & WAIS-I \\
\hline CTQ-SF total score & Rho $=-0.026, p=0.884$ & Rho $=-0.308, p=0.072$ & Rho $=-0.186, p=0.283$ \\
Emotional abuse & rho $=-0.078, p=0.656$ & Rho $=-0.087, p=0.619$ & Rho $=-0.125, p=0.476$ \\
Physical abuse & Rho $=0.035, p=0.842$ & Rho $=0.061, p=0.730$ & Rho $=-0.288, p=0.093$ \\
Sexual abuse & Rho $=0.035, p=0.842$ & Rho $=0.015, p=0.934$ & Rho $=0.087, p=0.619$ \\
Emotional neglect & Rho $=0.086, p=0.625$ & Rho $=-0.317, p=0.630$ & Rho $=-0.175, p=0.315$ \\
Physical neglect & Rho $=-0.460, p=0.791$ & Rho $=-0.232, p=0.180$ & Rho $=-0.099, p=0.572$ \\
MD score & Rho $=-0.034, p=0.844$ & Rho $=0.227, p=0.190$ & Rho $=0.063, p=0.720$ \\
\hline
\end{tabular}

\section{Exploratory Analysis}

As shown in Table 5, a higher test-retest reliability of CTQ-SF was shown in participants with a follow-up interval less than 12 months $(\mathrm{ICC}=0.853)$ compared to those with an interval longer than 12 months $(\mathrm{ICC}=0.504)$. The same trends of results (lower ICCs in those with a longer follow-up interval) were observed for all the subscales of CTQ-SF (see Table 5). 
Table 4 Correlations Between the CTQ-SF Scores and Other Clinical/Cognitive Assessments at Follow-Up, with Spearman's Rho and $p$ values Reported

\begin{tabular}{|l|c|c|c|}
\hline & PANSS & WAIS-DS & WAIS-I \\
\hline CTQ-SF total score & Rho $=0.089, p=0.613$ & Rho $=-0.196, p=0.258$ & Rho $=-0.028, p=0.875$ \\
Emotional abuse & Rho $=-0.108, p=0.538$ & $R h o=-0.232, p=0.180$ & Rho $=-0.181, p=0.299$ \\
Physical abuse & Rho $=-0.015, p=0.932$ & $R h o=-0.209, p=0.228$ & Rho $=-0.181, p=0.298$ \\
Sexual abuse & Rho $=-0.106, p=0.544$ & Rho $=0.157, p=0.367$ & Rho $=0.318, p=0.063$ \\
Emotional neglect & Rho $=0.086, p=0.625$ & Rho $=-0.002, p=0.990$ & Rho $=0.181, p=0.298$ \\
Physical neglect & Rho $=0.239, p=0.167$ & Rho $=0.326, p=0.056$ & Rho $=-0.307, p=0.073$ \\
MD score & Rho $=-0.061, p=0.729$ & Rho $=0.292, p=0.089$ & Rho $=0.057, p=0.745$ \\
\hline
\end{tabular}

Table 5 Exploratory Analysis on ICCs of the CTQ-SF Scores in Subsamples with Different Lengths of Follow-Up Intervals

\begin{tabular}{|l|l|l|}
\hline \multirow{2}{*}{ CTQ-SF Scores } & \multicolumn{2}{|c|}{ ICC (with 95\% Confidence Intervals) } \\
\cline { 2 - 3 } & Interval < I2 Months $(\mathbf{n}=\mathbf{2 3})$ & Interval $\geq$ I2 Months (n = I 2) \\
\hline Total score & $0.853^{*}(0.68,0.94)$ & $0.504^{*}(-0.10,0.83)$ \\
Emotional abuse & $0.890^{*}(0.76,0.95)$ & $0.488(-0.13,0.82)$ \\
Physical abuse & $0.713^{*}(0.44,0.87)$ & $0.724^{*}(0.31,0.91)$ \\
Sexual abuse & $0.885^{*}(0.75,0.95)$ & $0.584^{*}(0.02,0.86)$ \\
Emotional neglect & $0.659^{*}(0.35,0.84)$ & $0.224(-0.42,0.70)$ \\
Physical neglect & $0.827^{*}(0.63,0.92)$ & $0.544^{*}(0.03,0.84)$ \\
MD score & $0.294(-0.13,0.62)$ & $0.268(-0.33,0.72)$ \\
\hline
\end{tabular}

Notes: *Indicates significance at the 0.05 level.

\section{Discussion}

In this study, we investigated the long-term test-retest reliability of CTQ-SF in a sample of schizophrenia patients over a relatively long interval of 11 months on average. Generally, our results indicated excellent long-term test-retest reliability of the CTQ-SF total score as well as most of its subscales. Meanwhile, at both baseline and follow-up, no significant correlations were found between scores of the CTQ-SF and assessments of clinical symptoms or cognition. These results provide important evidence for validating the use of CTQ-SF in schizophrenia and may also point to its value in assessing other mental health populations.

As one of the most widely used assessments of childhood trauma in clinical research, ${ }^{12,19-23}$ previous studies have demonstrated that CTQ-SF is valid and has overall good longterm test-retest reliabilities in both healthy participants and multiple psychiatric populations. For example, in a study conducted by Cammack et al, ${ }^{43}$ the test-retest reliability of CTQSF was good when it was completed by 247 adult women twice with an interval between 18.1 and 369.9 weeks (ICCs $>0.7$ for all subscales). In another study conducted by Shannon et al, ${ }^{44}$ the CTQ-SF was highly reliable (ICCs $>0.8$ for 4 of the 5 subscales) when retested over 18 months in 60 patients with bipolar disorder. Clinical and neuroimaging studies have suggested the important role of childhood trauma in schizophrenia; $;^{5-8}$ however, little was known about the longterm test-retest reliability of CTQ-SF in schizophrenia patients. Our findings here may fill this knowledge gap by presenting the first investigation to our knowledge of CTQSF reliability in a long-term longitudinal schizophrenia population.

Stabilities of retrospective assessments, such as the CTQSF, generally rely on the accuracy of people's memories. ${ }^{45}$ Schizophrenia is known to be associated with certain types of psychopathology including cognitive impairments and delusional symptoms which may lead to memory distortions. ${ }^{46-48}$ Our results showed, however, that no significant relationships existed between the reported CTQ-SF scores and current cognitive functions or psychotic symptoms in patients with schizophrenia (Tables 3 and 4). We thus preliminarily concluded that CTQ-SF is a temporally stable and stateindependent assessment of childhood maltreatment in schizophrenic subjects, which is less likely to be influenced by patients' current clinical symptoms and cognitions.

Among five subscales of the CTQ-SF, the sexual abuse and emotional neglect subscales showed the highest and 
lowest test-retest reliability, respectively (Table 2). Our results are highly consist with a previous study conducted in healthy adults in the Netherlands: in such study, it was found that sexual abuse was most reliably reported during a 6-year follow-up; meanwhile, the emotional neglect subscale was found to be relatively unreliable, which may partly due to the impacts of participants' emotional states such as depression as proposed by the authors. ${ }^{49}$ This may also support a crosscultural generalizability of our results, indicating that contents of the emotional neglect dimension in CTQ-SF could be problematic in some ways. Nevertheless, the ICCs of all subscales were higher than 0.5 (Table 2), which suggest at least moderate reliability and generally consistent reporting. ${ }^{43}$

Similar to other studies, ${ }^{43,50,51}$ the assessments were done across varying follow-up intervals (ranging from 2 to 41 months) in the present study. To explore possible influences of interval length, we calculated ICCs in two subgroups with different intervals ( $<12$ months and $\geq 12$ months) separately, and lower ICCs were found in the subgroup with longer intervals (Table 5). This highlights the possibility that testretest reliability of the CTQ-SF might decrease over longer time intervals. However, we should remain cautious in drawing such a conclusion considering the small sample size $(\mathrm{n}=$ 12 only) and wide confidence intervals for the subgroup with intervals longer than 12 months (see Table 5).

Another issue to note is that although most published studies reporting on the CTQ's reliability did not mention the MD score, ${ }^{52-54}$ here we include the MD score in this study for its importance suggested by some recent work. ${ }^{35}$ We found that the MD score has a low test-retest reliability (ICC $<0.4$, Table 2). However, such a conclusion may need to be verified in a larger sample considering the wide confidence interval for the MD score's ICC (Table 2), too.

There are several minor points to note in the current study. First, as mentioned above, the sample size is relatively small and future studies are warranted to replicate out results in a larger sample. Second, during follow-up, patients were excluded when they had extensive declines in cognition and were unable to re-complete the CTQ-SF. This might cause a selection bias, and our conclusions may not be extrapolated to patients with poorer outcomes. Last, the follow-up interval is only 11 months on average in this study, and we are unable to know if the CTQ-SF results would be stable over a longer interval or even lifetime.

\section{Conclusion}

In conclusion, in this study, we investigated the long-term testretest reliability of the CTQ-SF in patients with schizophrenia over an interval of 11 months on average. The results suggested an excellent reliability of the CTQ-SF total score, and at least moderate reliabilities for all of its subscales. Moreover, no significant associations were found between the CTQ-SF and assessments of clinical symptoms or cognitions. Our results suggest that CTQ-SF is reliable to assess childhood trauma exposures in schizophrenia over relatively long time intervals, regardless of patients' current symptoms and cognitive status. However, future studies are warranted to replicate out results in a larger sample and to investigate whether the CTQ-SF would keep stable over longer time intervals.

\section{Ethics Statement}

The study was performed in accordance with the Declaration of Helsinki. The study was approved by the Ethics Committee of Second Xiangya Hospital in Changsha, and written informed consent was obtained from all participants or guardians.

\section{Acknowledgments}

This work was supported by the China Precision Medicine Initiative (grant number 2016YFC0906300) and the National Natural Science Foundation of China (grant numbers $81671335,81701325,81801353$ and 82071506). We thank Brendan Ross from McGill University for his excellent help in polishing the English language.

\section{Disclosure}

The authors declare that they have no conflicts of interest in this work.

\section{References}

1. McCutcheon RA, Reis Marques T, Howes OD. Schizophrenia - an overview. JAMA Psychiatry. 2020;77(2):201-210. doi:10.1001/ jamapsychiatry.2019.3360

2. Long Y, Ouyang X, Liu Z, et al. Associations among suicidal ideation, white matter integrity and cognitive deficit in first-episode schizophrenia. Front Psychiatry. 2018;9(Aug). doi:10.3389/fpsyt.2018.00391

3. Cao H, Zhou H, Cannon TD. Functional connectome-wide associations of schizophrenia polygenic risk. Mol Psychiatry. 2020. doi:10.1038/s41380-020-0699-3

4. Long Y, Liu Z, Chan CKY, et al. Altered temporal variability of local and large-scale resting-state brain functional connectivity patterns in schizophrenia and bipolar disorder. Front Psychiatry. 2020;11. doi:10.3389/fpsyt.2020.00422

5. Read J, Van Os J, Morrison AP, Ross CA. Childhood trauma, psychosis and schizophrenia: a literature review with theoretical and clinical implications. Acta Psychiatr Scand. 2005;112(5):330-350. doi:10.1111/j.1600-0447.2005.00634.x

6. Bonoldi I, Simeone E, Rocchetti M, et al. Prevalence of self-reported childhood abuse in psychosis: a meta-analysis of retrospective studies. Psychiatry Res. 2013;210(1):8-15. doi:10.1016/j.psychres.201 3.05 .003 
7. Aas M, Haukvik UK, Djurovic S, et al. BDNF val66met modulates the association between childhood trauma, cognitive and brain abnormalities in psychoses. Prog Neuropsychopharmacol Biol Psychiatry. 2013;46:181-188. doi:10.1016/j.pnpbp.2013.07.008

8. Aas M, Haukvik UK, Djurovic S, et al. Interplay between childhood trauma and BDNF val66met variants on blood BDNF mRNA levels and on hippocampus subfields volumes in schizophrenia spectrum and bipolar disorders. J Psychiatr Res. 2014;59:14-21. doi:10.1016/j. jpsychires.2014.08.011

9. Popovic D, Ruef A, Dwyer DB, et al. Traces of trauma: a multivariate pattern analysis of childhood trauma, brain structure, and clinical phenotypes. Biol Psychiatry. 2020;88(11):829-842. doi:10.1016/j.biopsych.2020.05.020

10. Cancel A, Comte M, Truillet R, et al. Childhood neglect predicts disorganization in schizophrenia through grey matter decrease in dorsolateral prefrontal cortex. Acta Psychiatr Scand. 2015;132 (4):244-256. doi:10.1111/acps.12455

11. Cisler JM. Childhood trauma and functional connectivity between amygdala and medial prefrontal cortex: a dynamic functional connectivity and large-scale network perspective. Front Syst Neurosci. 2017;11. doi:10.3389/fnsys.2017.00029

12. Huang D, Liu Z, Cao H, Yang J, Wu Z, Long Y. Childhood trauma is linked to decreased temporal stability of functional brain networks in young adults. J Affect Disord. 2021;290:23-30. doi:10.1016/j. jad.2021.04.061

13. Quidé Y, O’Reilly N, Rowland JE, Carr VJ, Elzinga BM, Green MJ. Effects of childhood trauma on working memory in affective and non-affective psychotic disorders. Brain Imaging Behav. 2017;11 (3):722-735. doi:10.1007/s11682-016-9548-Z

14. Quidé Y, Ong XH, Mohnke S, et al. Childhood trauma-related alterations in brain function during a theory-of-mind task in schizophrenia Schizophr Res. 2017;189:162-168. doi:10.1016/j.schres.2017.02.012

15. Aas M, Kauppi K, Brandt CL, et al. Childhood trauma is associated with increased brain responses to emotionally negative as compared with positive faces in patients with psychotic disorders. Psychol Med. 2017;47(4):669-679. doi:10.1017/S0033291716002762

16. Cancel A, Comte M, Boutet C, et al. Childhood trauma and emotional processing circuits in schizophrenia: a functional connectivity study. Schizophr Res. 2017;184:69-72. doi:10.1016/j.schres.201 6.12 .003

17. Popovic D, Schmitt A, Kaurani L, et al. Childhood trauma in schizophrenia: current findings and research perspectives. Front Neurosci. 2019;13. doi:10.3389/fnins.2019.00274

18. Bernstein DP, Stein JA, Newcomb MD, et al. Development and validation of a brief screening version of the childhood trauma questionnaire. Child Abus Negl. 2003;27(2):169-190. doi:10.1016/ S0145-2134(02)00541-0

19. Kim D, Park SC, Yang H, Oh DH. Reliability and validity of the Korean version of the childhood trauma questionnaire-short form for psychiatric outpatients. Psychiatry Investig. 2011;8(4):305-311. doi:10.4306/pi.2011.8.4.305

20. Fawzi MH, Fawzi MM, Fouad AA. Parent abuse by adolescents with first-episode psychosis in egypt. $J$ Adolesc Health. 2013;53 (6):730-735. doi:10.1016/j.jadohealth.2013.07.004

21. Wang Z, Xue Z, Pu W, et al. Comparison of first-episode and chronic patients diagnosed with schizophrenia: symptoms and childhood trauma. Early Interv Psychiatry. 2013;7(1):23-30. doi:10.1111/ j.1751-7893.2012.00387.x

22. Ju Y, Wang M, Lu X, et al. The effects of childhood trauma on the onset, severity and improvement of depression: the role of dysfunctional attitudes and cortisol levels. $J$ Affect Disord. 2020;276:402-410. doi:10.1016/j.jad.2020.07.023

23. Zhang CH, Li G, Fan ZY, Tang XJ, Zhang F. Psychological capital mediating the relationship between childhood trauma and alexithymia in Chinese medical students: a cross-sectional study. Psychol Res Behav Manag. 2020;13:1343-1352. doi:10.2147/PRBM.S288647
24. Jiang WJ, Zhong BL, Liu LZ, Zhou YJ, Hu XH, Li Y. Reliability and validity of the Chinese version of the childhood trauma questionnaire-short form for inpatients with schizophrenia. PLoS One. 2018;13(12):e0208779. doi:10.1371/journal.pone.0208779

25. Kim D, Bae H, Han C, Oh HY, MacDonald K. Psychometric properties of the Childhood Trauma Questionnaire-Short Form (CTQ-SF) in Korean patients with schizophrenia. Schizophr Res. 2013;144(13):93-98. doi:10.1016/j.schres.2012.12.020

26. Fisher HL, Craig TK, Fearon P, et al. Reliability and comparability of psychosis patients' retrospective reports of childhood abuse. Schizophr Bull. 2011;37(3):546-553. doi:10.1093/schbul/sbp103

27. Halverson CE. Remembering your parents: reflections on the retrospective method. J Pers. 1988;56(2):435-443. doi:10.1111/j.14676494.1988.tb00895.x

28. Piolino P, Desgranges B, Benali K, Eustache F. Episodic and semantic remote autobiographical memory in ageing. Memory. 2002;10 (4):239-257. doi:10.1080/09658210143000353

29. Lewinsohn PM, Rosenbaum M. Recall of parental behavior by acute depressives, remitted depressives, and nondepressives. J Pers Soc Psychol. 1987;52(3):611-619. doi:10.1037/0022-3514.52.3.611

30. Feldman-Summers S, Pope KS. The experience of "forgetting" childhood abuse: a national survey of psychologists. $J$ Consult Clin Psychol. 1994;62(3):636-639. doi:10.1037/0022006X.62.3.636

31. Gerlsma C, Emmelkamp PMG, Arrindell WA. Anxiety, depression, and perception of early parenting: a meta-analysis. Clin Psychol Rev. 1990;10(3):251-277. doi:10.1016/0272-7358(90)90062-F

32. Olsen RA. A review of the seven sins of memory: how the mind forgets and remembers. J Psychol Finance Mark. 2001;2(3):174. doi:10.1207/s15327760jpfm0203_8

33. Kjellsson G, Clarke P, Gerdtham UG. Forgetting to remember or remembering to forget: a study of the recall period length in health care survey questions. $J$ Health Econ. 2014;35(1):34-46. doi:10.1016/j.jhealeco.2014.01.007

34. Keating XD, Zhou K, Liu X, et al. Reliability and concurrent validity of global physical activity questionnaire (GPAQ): a systematic review. Int $J$ Environ Res Public Health. 2019;16(21):21. doi: $10.3390 /$ ijerph 16214128

35. MacDonald K, Thomas ML, Sciolla AF, et al. Minimization of childhood maltreatment is common and consequential: results from a large, multinational sample using the childhood trauma questionnaire. PLoS One. 2016;11:1. doi:10.1371/journal.pone.0146058

36. Kay SR, Fiszbein A, Opler LA. The positive and negative syndrome scale (PANSS) for schizophrenia. Schizophr Bull. 1987;13 (2):261-276. doi:10.1093/schbul/13.2.261

37. Yao-xian G. Revision of wechsler's adult intelligence scale in China. Acta Psychol Sin. 1983;15(03):121-129.

38. Tan W, Liu Z, Xi C, et al. Decreased integration of the frontoparietal network during a working memory task in major depressive disorder. Aust N Z J Psychiatry. 2021;55(6):577-587. doi:10.1177/00048674 20978284

39. Long Y, Chen C, Deng M, et al. Psychological resilience negatively correlates with resting-state brain network flexibility in young healthy adults: a dynamic functional magnetic resonance imaging study. Ann Transl Med. 2019;7(24):809. doi:10.21037/atm.2019.12.45

40. Shrout PE, Fleiss JL. Intraclass correlations: uses in assessing rater reliability. Psychol Bull. 1979;86(2):420-428. doi:10.1037/00332909.86.2.420

41. Cicchetti DV. Guidelines, criteria, and rules of thumb for evaluating normed and standardized assessment instruments in psychology. Psychol Assess. 1994;6(4):284-290. doi:10.1037/10403590.6.4.284

42. Cao H, McEwen SC, Forsyth JK, et al. Toward leveraging human connectomic data in large consortia: generalizability of fmri-based brain graphs across sites, sessions, and paradigms. Cereb Cortex. 2019;29(3):1263-1279. doi:10.1093/cercor/bhy032 
43. Cammack AL, Hogue CJ, Drews-Botsch CD, et al. Test-retest reliability of retrospective self-reported maternal exposure to childhood abuse and neglect. Arch Womens Ment Health. 2016;19(2):415-421. doi:10.1007/s00737-015-0536-x

44. Shannon C, Hanna D, Tumelty L, et al. Reliability of reports of childhood trauma in bipolar disorder: a test-retest study over 18 months. J Trauma Dissociation. 2016;17(4):511-519. doi:10.1080/ 15299732.2016.1141147

45. Paivio SC. Stability of retrospective self-reports of child abuse and neglect before and after therapy for child abuse issues. Child Abus Negl. 2001;25(8):1053-1068. doi:10.1016/S0145-2134(01)00256-3

46. Aleman A, Hijman R, De Haan EHF, Kahn RS. Memory impairment in schizophrenia: a meta-analysis. Am J Psychiatry. 1999;156 (9):1358-1366. doi:10.1176/ajp.156.9.1358

47. Bhatt R, Laws KR, McKenna PJ. False memory in schizophrenia patients with and without delusions. Psychiatry Res. 2010;178 (2):260-265. doi:10.1016/j.psychres.2009.02.006

48. Koller WN, Cannon TD. Paranoia is associated with impaired novelty detection and overconfidence in recognition memory judgments. J Abnorm Psychol. 2021;130(3):273-285. doi:10.1037/abn0000664

49. Wielaard I, Stek ML, Comijs HC, Rhebergen D. Reliability of retrospective reports on childhood abuse and its determinants in older adults during a 6-year follow-up. J Psychiatr Res. 2018;105:9-16. doi:10.1016/j.jpsychires.2018.08.009
50. Alioto AG, Kramer JH, Borish S, et al. Long-term test-retest reliability of the California verbal learning test-second edition. Clin Neuropsychol. 2017;31(8):1449-1458. doi:10.1080/13854046.2017.1310300

51. Yu L, Zhu X, Shek DTL, Zou XB, Deng HZ, Au Yeung PWH. Validation of the simplified Chinese psychoeducational profile third edition in Mainland China. J Autism Dev Disord. 2019;49 (4):1599-1612. doi:10.1007/s10803-018-3827-z

52. Li XB, Liu JT, Zhu XZ, Zhang L, Tang YL, Wang CY. Childhood trauma associates with clinical features of bipolar disorder in a sample of Chinese patients. J Affect Disord. 2014;168:58-63. doi:10.1016/j.jad.2014.06.017

53. Grassi-Oliveira R, Cogo-Moreira H, Salum GA, et al. Childhood Trauma Questionnaire (CTQ) in Brazilian samples of different age groups: findings from confirmatory factor analysis. PLoS One. 2014;9:1. doi:10.1371/journal.pone.0087118

54. Garrusi B, Nakhaee N. Validity and reliability of a Persian version of the childhood trauma questionnaire. Psychol Rep. 2009;104 (2):509-516. doi:10.2466/PR0.104.2.509-516
Psychology Research and Behavior Management

\section{Publish your work in this journal}

Psychology Research and Behavior Management is an international, peer-reviewed, open access journal focusing on the science of psychology and its application in behavior management to develop improved outcomes in the clinical, educational, sports and business arenas. Specific topics covered in the journal include: Neuroscience, memory and decision making; Behavior modification and management; Clinical
Dovepress

applications; Business and sports performance management; Social and developmental studies; Animal studies. The manuscript management system is completely online and includes a very quick and fair peer-review system, which is all easy to use. Visit http://www. dovepress.com/testimonials.php to read real quotes from published authors. 